\title{
Testing particle trapping in transition disks with ALMA ${ }^{\star}$
}

\author{
P. Pinilla ${ }^{1}$, N. van der Marel $^{1}$, L. M. Pérez ${ }^{2, \star \star}$, E. F. van Dishoeck ${ }^{1,3}$, S. Andrews ${ }^{4}$, T. Birnstiel ${ }^{4}$, \\ G. Herczeg ${ }^{5}$, K. M. Pontoppidan ${ }^{6}$, and T. van Kempen ${ }^{1}$ \\ ${ }^{1}$ Leiden Observatory, Leiden University, PO Box 9513, 2300 RA Leiden, The Netherlands \\ e-mail: pinilla@strw. leidenuniv.nl \\ 2 National Radio Astronomy Observatory, PO Box O, Socorro, NM 87801, USA \\ 3 Max-Planck-Institut für Extraterrestrische Physik, Giessenbachstrasse 1, 85748 Garching, Germany \\ ${ }^{4}$ Harvard-Smithsonian Center for Astrophysics, 60 Garden Street, Cambridge, MA 02138, USA \\ 5 Kavli Institute for Astronomy and Astrophysics, Peking University, Yi He Yuan Lu 5, Haidian District, 100871 Beijing, PR China \\ ${ }^{6}$ Space Telescope Science Institute, 3700 San Martin Drive, Baltimore, MD 21218, USA
}

Received 2 June 2015 / Accepted 8 September 2015

\begin{abstract}
Some protoplanetary disks show evidence of inner dust cavities. Recent observations of gas and dust of these so-called transition disks support the hypothesis that these cavities originate from particle trapping in pressure bumps. We present new Atacama Large Millimeter/submillimeter Array (ALMA) continuum observations at $336 \mathrm{GHz}$ of two transition disks, SR21 and HD 135344B. In combination with previous ALMA observations from Cycle 0 at $689 \mathrm{GHz}$, we compare the visibility profiles at the two frequencies and calculate the spectral index $\left(\alpha_{\mathrm{mm}}\right)$. The observations of SR 21 show a clear shift in the visibility nulls, indicating radial variations of the inner edge of the cavity at the two wavelengths. Notable radial variations of the spectral index are also detected for SR 21 with values of $\alpha_{\mathrm{mm}} \sim 3.8-4.2$ in the inner region $\left(r \lesssim 35 \mathrm{AU}\right.$ ) and $\alpha_{\mathrm{mm}} \sim 2.6-3.0$ outside. An axisymmetric ring (which we call the ring model) or a ring with the addition of an azimuthal Gaussian profile, for mimicking a vortex structure (which we call the vortex model), is assumed for fitting the disk morphology. For SR 21, the ring model better fits the emission at $336 \mathrm{GHz}$, conversely the vortex model better fits the $689 \mathrm{GHz}$ emission. For HD 135344B, neither a significant shift in the null of the visibilities nor radial variations of $\alpha_{\mathrm{mm}}$ are detected. Furthermore, for HD 135344B, the vortex model fits both frequencies better than the ring model. However, the azimuthal extent of the vortex increases with wavelength, contrary to model predictions for particle trapping by anticyclonic vortices. For both disks, the azimuthal variations of $\alpha_{\mathrm{mm}}$ remain uncertain to confirm azimuthal trapping. The comparison of the current data with a generic model of dust evolution that includes planet-disk interaction suggests that particles in the outer disk of SR 21 have grown to millimetre sizes and have accumulated in a radial pressure bump, whereas with the current resolution there is not clear evidence of radial trapping in HD 135344B, although it cannot be excluded either.
\end{abstract}

Key words. protoplanetary disks - circumstellar matter - planets and satellites: formation

\section{Introduction}

Recent observations of transition disks (disks with inner dust cavities) suggest that their structures may originate from particle trapping in pressure bumps (e.g. van der Marel et al. 2013; Zhang et al. 2014). Particle accumulation in pressure maxima has been suggested to solve the problem of rapid inwards drift of particles (e.g. Weidenschilling 1977; Brauer et al. 2008), implying that planetary embryos may form in localised environments (e.g. Klahr \& Henning 1997; Johansen et al. 2007). Pressure bumps may occur because of the presence of one or multiple planets in the disk (e.g. Dodson-Robinson \& Salyk 2011; Pinilla et al. 2012, 2015; Zhu et al. 2012), but other phenomena, such as dead zones may also create pressure traps and explain their structures (e.g. Regály et al. 2011; Flock et al. 2015). Observations of transition disks reveal that dust cavities appear to be smaller at shorter wavelengths (e.g. Dong et al. 2012; Garufi et al. 2013). This spatial segregation of small and $\mathrm{mm}$-sized grains is a natural consequence of particle trapping

\footnotetext{
* Appendices are available in electronic form at http: //www . aanda.org

$\star \star$ Jansky Fellow.
}

in the planet-disk interaction scenario (e.g. Rice et al. 2006; de Juan Ovelar et al. 2013).

The spectral index $\alpha_{\mathrm{mm}}\left(F_{v} \propto v^{\alpha_{\mathrm{mm}}}\right)$ provides information about the particle size in protoplanetary disks (see Testi et al. 2014, for a review). For (sub-) micron-sized dust, as found in the interstellar medium, $\alpha_{\mathrm{mm}}$ is expected to have values of $\gtrsim 3.5-4.0$ (e.g. Finkbeiner et al. 1999). When dust grows to millimetre sizes, $\alpha_{\mathrm{mm}}$ is expected to decrease (Draine 2006; Ricci et al. 2010). Radial increases in $\alpha_{\mathrm{mm}}$ (on 100 AU scales) have been found for individual disks without cavities (e.g. Guilloteau et al. 2011; Pérez et al. 2012), consistent with radial drift. In contrast, the inner region of transition disks is depleted of large grains, so that $\alpha_{\mathrm{mm}}$ would decrease with radius from the central star (Pinilla et al. 2014). However, these radial variations for $\alpha_{\mathrm{mm}}$ in transition disk have not been spatially resolved to date.

In this paper, we combine observations from ALMA Cycle 0 at $689 \mathrm{GHz}(\sim 450 \mu \mathrm{m})$ and Cycle 1 at $336 \mathrm{GHz}(\sim 870 \mu \mathrm{m})$ of SR 21 and HD 135344B (also known as SAO 206462) to compare the morphological structures at the two frequencies and calculate $\alpha_{\mathrm{mm}}$ throughout the disk. In addition, we compare the results with a generic model of particle trapping and dust growth 

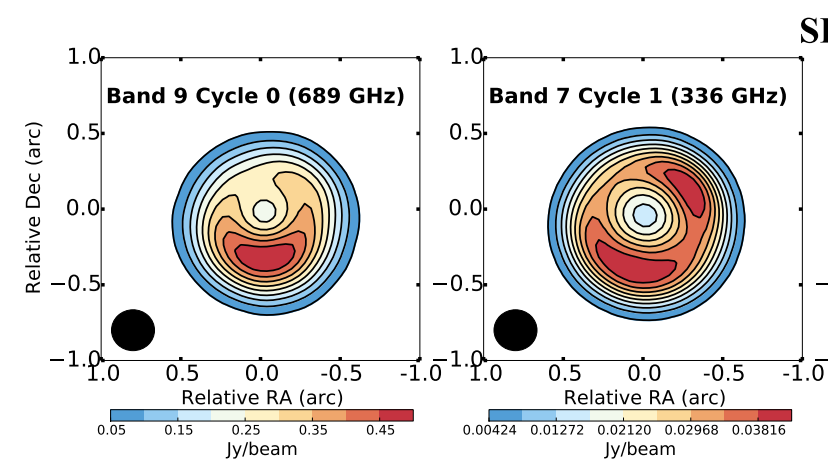

SR 21
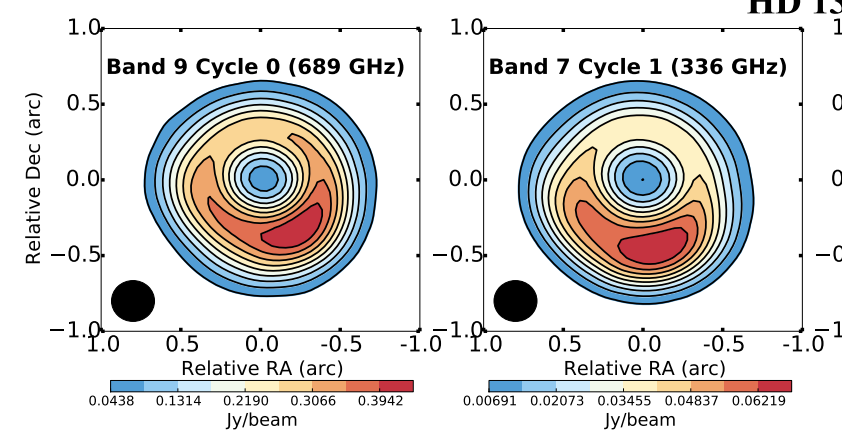

HD 135344B

Fig. 1. ALMA observations of dust continuum emission for SR 21 (top panels) and HD 135344B (bottom panels). The left columns show the Band $9(689 \mathrm{GHz})$ and Band $7(336 \mathrm{GHz})$ continuum, with the contour overlaid at $10,20, \ldots, 90 \%$ of the peak. The third column shows the overlay of the contours with a step of $20 \%$ of the peak (black is Band 9, red is Band 7). The right column shows the resolved $\alpha_{\mathrm{mm}}$ in colour contours. The beam size is indicated in each plot.

in a pressure bump induced by an embedded planet, and place the results in the context of other transition disks.

\section{Targets and observations}

SR 21 is a G3 star located in the Ophiuchus star-forming region at a distance of $d=120 \mathrm{pc}$ (Loinard et al. 2008). The disk was identified as a transition disk by its mid-infrared SED, with a cavity radius of $\sim 18 \mathrm{AU}$ (Brown et al. 2007). Observations with the Submillimeter Array (SMA) at $850 \mu \mathrm{m}$ confirmed a $235 \mathrm{AU}$ cavity and suggested an azimuthal disk asymmetry (Brown et al. 2009; Andrews et al. 2011), which was much more clearly seen with ALMA observations in Cycle 0 (Pérez et al. 2014). While $450 \mu \mathrm{m}$ imaging with ALMA shows strong depletion of large mm-sized grains, by a factor of $10^{3}$ or more in the inner region (van der Marel et al. 2015a), $H$-band scattered light imaging indicates that the small grains are much less depleted, perhaps by as little as a factor of 10 (Follette et al. 2013).

HD 135344B is an F4 star located in the Sco OB2-3 starforming region at a distance of $d=140 \mathrm{pc}$ (van Boekel et al. 2005). A cavity radius of $\sim 45 \mathrm{AU}$ was also identified by its SED (Brown et al. 2007) and confirmed by SMA observations (Brown et al. 2009). One of the most intriguing characteristics of this disk is its spiral arms observed in scattered light images (Muto et al. 2012; Garufi et al. 2013). The observations with VLT/NACO did not show signs of a gap in small dust grains down to 28 AU radius (Garufi et al. 2013).

Our observations are from ALMA Cycle 0 program 2011.0.00724.S (P.I. Pérez) and Cycle 1 program 2012.1.00158.S (P.I. van Dishoeck), taken in Band 9 and Band 7, respectively. The details of the calibration are described in Pérez et al. (2014) and van der Marel et al. (2015c). For both observation sets, the images were obtained using Briggs weighting with a robust parameter of 0.5 and and the
Table 1. Observation properties.

\begin{tabular}{cccccccc}
\hline \hline Target & Band & $\begin{array}{c}v \\
(\mathrm{GHz})\end{array}$ & $\begin{array}{c}\Delta v \\
(\mathrm{GHz})\end{array}$ & $\begin{array}{c}F_{\text {peak }} \\
(\mathrm{mJy})\end{array}$ & $\begin{array}{c}F_{\text {total }} \\
(\mathrm{mJy})\end{array}$ & $\begin{array}{c}\sigma \\
(\mathrm{mJy})\end{array}$ & Date \\
\hline SR 21 & 7 & 336 & 3.2 & 42.4 & 349 & 0.08 & $26 / 07 / 2014$ \\
& 9 & 689 & 7.5 & 500 & 2877 & 2.0 & $18 / 07 / 2012$ \\
\hline HD 135344B & 7 & 336 & 3.2 & 69.1 & 636 & 0.13 & $27 / 07 / 2014$ \\
& 9 & 689 & 7.5 & 438 & 3360 & 2.5 & $05 / 07 / 2012$ \\
\hline
\end{tabular}

beam is set to be the same at both bands by the post-processing of the images, which is the best possible beam size corresponding to both the Band 7 and Band 9 observations $\left(0.27^{\prime \prime}\right)$. The details of the images are given in Table 1.

As the data are taken almost two years apart, the centre of the 2014 data was shifted before overlaying the images to compensate for the proper motion of the stars. The proper motion is -12 ,

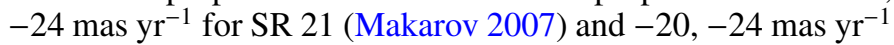
for HD 135344B (Høg et al. 2000). Figure 1 shows the continuum maps and their overlay. The shape and contrast of the asymmetry look similar between Band 7 and 9 for HD 135344B. In contrast, SR 21 looks more symmetric in Band 7. There is an azimuthal shift in the continuum overlay for both sources. The position of the gain calibrator (the same for both observations) shifts $\lesssim 0.1$ mas, meaning that the azimuthal shift is not due to calibration. With respect to the SMA images in 2006/2007 (Brown et al. 2009), the asymmetries are also shifted.

\section{Data analysis}

\subsection{Visibilities and disk morphology}

The real part of the visibilities at both frequencies is shown in Fig. 2. These are extracted from the continuum data and deprojected using $i=15^{\circ}$ and position angle $\mathrm{PA}=14^{\circ}$ for SR 21, 


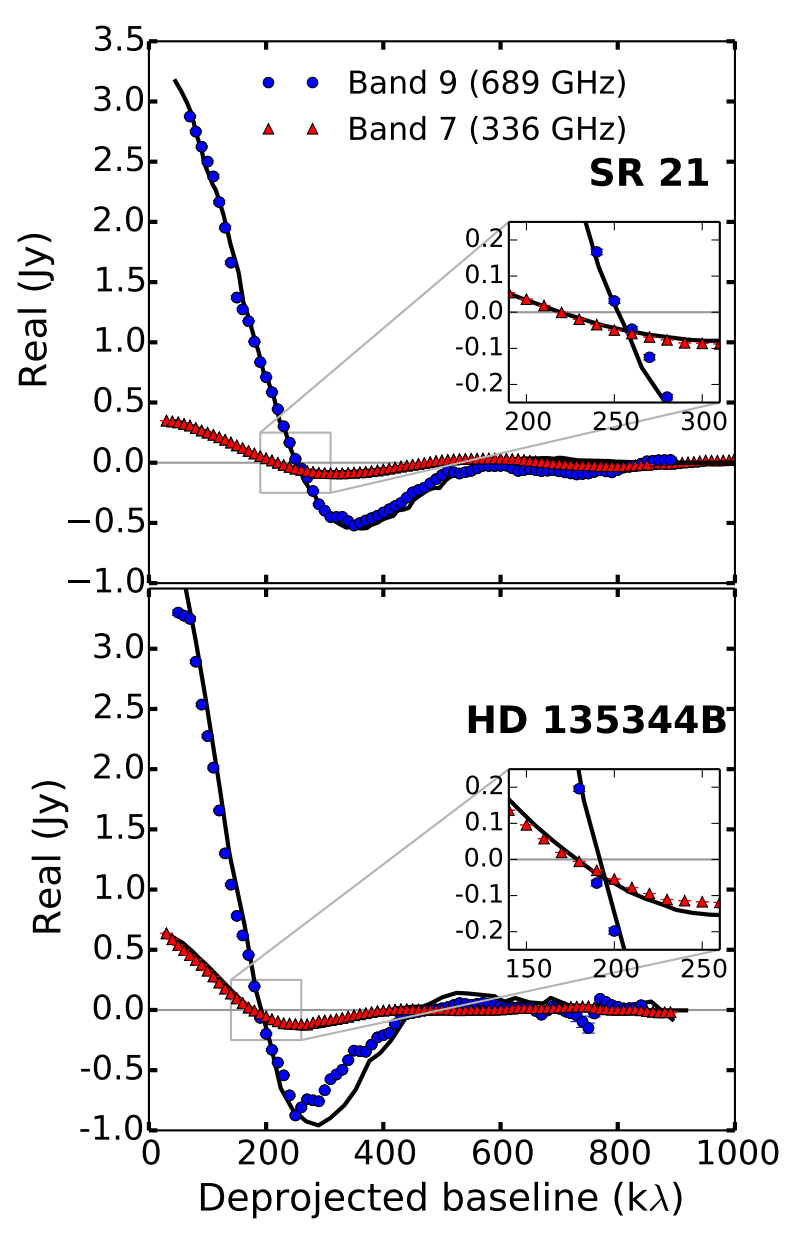

Fig. 2. Real part of the visibilities at $689 \mathrm{GHz}(\sim 450 \mu \mathrm{m}$, Band 9) and at $336 \mathrm{Gz}(\sim 870 \mu \mathrm{m}$, Band 7) for SR 21 (top panel) and HD 135344B (bottom panel). The plot includes error bars that are of the size of the plotting points. At both wavelengths, the models with the best-fit parameters are over-plotted (solid line), which is the ring model in the case of SR 21, and the vortex model for HD 135344B.

and $i=20^{\circ}$ and $\mathrm{PA}=63^{\circ}$, as derived in Pontoppidan et al. (2008) and van der Marel et al. (2015a). The data are binned by taking the mean of the available data points in bins of $20 \mathrm{k} \lambda$, with a minimum of five data points per bin. In Fig. 2, we show the real part of the visibilities for the two bands. The length of the projected baseline where the visibilities cross zero (the null) indicates the location inner edge of the cavity in a given bandpass (smaller values for the null mean that the cavity inner edge is further out for the same disk properties). The nulls for SR 21 are $\sim 220 \mathrm{k} \lambda$ at Band 9 and $\sim 250 \mathrm{k} \lambda$ at Band 7. For HD 135344B the null is almost at the same position at both wavelengths $(\sim 190 \mathrm{k} \lambda)$. The imaginary part of the visibilities are presented in Appendix A.

To fit the visibility profiles and constrain the structure of both disks, we used the same morphological models described in Pérez et al. (2014). One model is a ring-like emission described by $F(r, \theta)=F_{R} \mathrm{e}^{-\left(r-r_{R}\right)^{2} / 2 \sigma_{R}^{2}}$, where $r_{R}$ is the location of the peak of the ring emission, $F_{R}$ the flux density at $r_{R}$, and $\sigma_{R}$ the ring width (ring model). The other model assumes a ring together with an azimuthal Gaussian profile to mimic a vortex structure (Lyra \& Lin 2013). The vortex is described by $F(r, \theta)=F_{V} \mathrm{e}^{-\left(r-r_{V}\right)^{2} / 2 \sigma_{r, V}^{2}} \mathrm{e}^{-\left(\theta-\theta_{V}\right)^{2} / 2 \sigma_{\theta, V}^{2}}$, with $r_{V}$ and $\theta_{V}$ being the radius and PA at the peak of the vortex, $F_{V}$ the flux density at $\left(r_{V}, \theta_{V}\right)$, and $\sigma_{\theta, V}, \sigma_{r, V}$ is the width of the vortex in the radial and azimuthal direction respectively (vortex model). The parameters of the best-fit model are found by minimising $\chi^{2}$ using the same Markov chain Monte Carlo (MCMC) approach implemented by Pérez et al. (2014). The results are summarised in Table 2. The errors from the MCMC calculations are much smaller than the spatial uncertainty from the observations, which is typically $\sim 10 \%$ of the beam size (i.e. $\sim 3$ AU for SR 21 and $\sim 4$ for HD 135344B) for the high signal to noise of the data (Table 1). For the MCMC calculations, the inclination and PA are fixed to the values found by Pérez et al. (2014) for both disks. The model including a vortex did not converge to a physical solution for SR 21 in Band 7, because the azimuthal extension of the vortex covers more than $2 \pi$. In summary, the SR 21 data are best fit with a ring model, while the HD 135344B data are best fit with a vortex model (see Fig. 2). The residuals obtained by subtracting the best-fit model from the data in Band 7 are shown for both targets in Appendix B.

In the case of SR 21, the best-fit model indicates that larger grains, as traced at longer wavelengths by Band 7, are more spatially confined than the smaller grains traced at shorter wavelengths by Band $9\left(\sigma_{R} \sim 12 \mathrm{AU}\right.$ at $870 \mu \mathrm{m}$ vs. $\sigma_{R} \sim 15 \mathrm{AU}$ at $450 \mu \mathrm{m})$. Furthermore, the peak of emission in the ring is at larger radii in Band 7 than in Band $9\left(r_{R} \sim 41 \mathrm{AU}\right.$ at $870 \mu \mathrm{m}$ vs. $r_{R} \sim 36 \mathrm{AU}$ at $\left.450 \mu \mathrm{m}\right)$. The location of the inner edge of the cavity at the two frequencies varies from $\sim 29 \mathrm{AU}$ in Band 9 to $\sim 35 \mathrm{AU}$ in Band 7, which together with the wider ring, is consistent with the shift of the null of the visibilities (Fig. 2). For HD 135344B, the vortex model is consistently the best-fit model for both wavelengths. The inner edge of the cavity is almost constant in the two bands, as shown in the visibility nulls (Fig. 2). However, the width of the vortex in both the radial and azimuthal directions is significantly larger in Band 7, and its aspect ratio $\left(\sigma_{\theta, V} / \sigma_{r, V}\right)$ decreases from 7.1 in Band 9 to 3.3 in Band 7.

\subsection{Spectral index}

The position-dependent spectral index (right panels of Fig. 1) is calculated using both bands as $\alpha_{\mathrm{mm}}=\ln \frac{F_{v_{B 7}}}{F_{v_{B 9}}} / \ln \frac{v_{B 7}}{v_{B 9}}$. All data with $F<5 \sigma$ are excluded. The dust emission within the cavity is detected at a high level of significance $(S / N \sim 320 \sigma$ and $\sim 250 \sigma$ in Band 7, and $\sim 120 \sigma$ and $\sim 80 \sigma$ in Band 9, for SR 21 and HD $135344 \mathrm{~B}$, respectively), so $\alpha_{\mathrm{mm}}$ can be accurately computed in the cavity separately from the dust ring. The uncertainty of $\alpha_{\mathrm{mm}}$ is calculated with error propagation from the observational uncertainty, which includes the calibration uncertainties $(\sim 20 \%$ and $\sim 10 \%$ in Band 9 and 7 , respectively) and the noise level or rms $(\sigma)$ of the observations. With the high signal-to-noise of the data, the uncertainty in the spectral index $\sigma_{\alpha_{\mathrm{mm}}}$ at a given position is dominated by the calibration uncertainties and approximates to $\approx\left(\ln v_{1} / v_{2}\right)^{-1} \sqrt{\left(0.2^{2}+0.1^{2}\right)} \approx 0.3$, in both cases. However, for the relative spatial changes in $\alpha_{\mathrm{mm}}$, the systematic calibration uncertainty does not contribute since it is constant across the field, and changes in $\alpha_{\mathrm{mm}}$ inside and outside the dust cavity have high significance with maximum values of $\sigma_{\alpha_{\mathrm{mm}}}$ of $\approx 0.01$. SR 21 shows significant radial variations of $\alpha_{\mathrm{mm}}$, with values of $\alpha_{\mathrm{mm}} \sim 3.8-4.2$ within $\sim 0.3^{\prime \prime}$ radius $(\sim 35 \mathrm{AU})$ and $\alpha_{\mathrm{mm}} \sim 2.6-3.0$ outside $\left(\Delta \alpha_{\mathrm{mm}} \gtrsim 120 \sigma_{\alpha_{\mathrm{mm}}}\right)$. This implies a lack of mm-grains in the inner region $(r \lesssim 35 \mathrm{AU})$, and is consistent with accumulation of $\mathrm{mm}$-grains in a localised region in the outer disk. For HD 135344B, no significant radial variations for $\alpha_{\mathrm{mm}}$ are found, and the values remain within a range of 2.6-3.2. Although Fig. 1 shows azimuthal variations of $\alpha_{\mathrm{mm}}$ for both 
Table 2. Best-fit parameters the disk morphology models of ring or ring+vortex.

(a) Ring model

\begin{tabular}{c|ccccc}
\hline \hline Target & $\begin{array}{c}v \\
(\mathrm{GHz})\end{array}$ & $\chi^{2}$ & $\begin{array}{c}F_{R} \\
(\mu \mathrm{m})\end{array}$ & $\begin{array}{c}r_{R} \\
(\mathrm{AU})\end{array}$ & $\begin{array}{c}\sigma_{R} \\
(\mathrm{AU})\end{array}$ \\
\hline \multirow{2}{*}{ SR 21 } & 336 & 2.07 & 0.71 & 41 & 12 \\
& 689 & 1.39 & 5.91 & 36 & 15 \\
\hline \multirow{2}{*}{ HD 135344B } & 336 & 3.75 & 0.84 & 61 & 19 \\
& 689 & 1.18 & 5.72 & 60 & 18 \\
\hline
\end{tabular}

(b) Ring+Vortex model

\begin{tabular}{c|cccccccccc}
\hline \hline Target & $\begin{array}{c}v \\
(\mathrm{GHz})\end{array}$ & $\chi^{2}$ & $\begin{array}{c}F_{R} \\
(\mu \mathrm{m})\end{array}$ & $\begin{array}{c}r_{R} \\
(\mathrm{AU})\end{array}$ & $\begin{array}{c}\sigma_{R} \\
(\mathrm{AU})\end{array}$ & $\begin{array}{c}F_{V} \\
(\mu \mathrm{m})\end{array}$ & $\begin{array}{c}r_{V} \\
(\mathrm{AU})\end{array}$ & $\begin{array}{c}\theta_{V} \\
\left({ }^{\circ}\right)\end{array}$ & $\begin{array}{c}\sigma_{R, V} \\
(\mathrm{AU})\end{array}$ & $\begin{array}{c}\sigma_{\theta, V} \\
(\mathrm{AU})\end{array}$ \\
\hline SR 21 & 689 & 1.06 & 4.72 & 35 & 14 & 4.0 & 46 & 178 & 14 & 40 \\
\hline \multirow{2}{*}{ HD 135344B } & 336 & 1.52 & 0.70 & 70 & 14 & 0.88 & 43 & 172 & 16 & 53 \\
& 689 & 1.05 & 5.24 & 65 & 16 & 7.0 & 42 & 194 & 7.0 & 47 \\
\hline
\end{tabular}

Notes. All data of Band 9 are identical than Pérez et al. (2014). The parameters for SR 21 in Band 7 of the ring+vortex model are omitted because of the unphysical results. The errors from the MCMC calculations are much smaller than the spatial uncertainty from the observations (and therefore omitted), which is $\sim 10 \%$ of the beam size (i.e. $\sim 3$ AU for SR 21 and $\sim 4$ AU for HD 135344B).

sources, these depend considerably on how the two images are overlaid in the calculation of $\alpha_{\mathrm{mm}}$ (see Appendix C), and therefore any azimuthal variation within a range of $\alpha_{\mathrm{mm}} \simeq 2.6-3.2$ remains uncertain.

\section{Theoretical predictions of particle trapping}

Dust trapping, in radial and azimuthal pressure bumps, depends on the coupling of the particles to the gas, pressure gradient, and disk turbulence. The dimensionless stopping time, Stokes number, quantifies the coupling of the particles and it is defined in the mid-plane (and assuming a Gaussian vertical profile for the gas density) as $S t=a \rho_{\mathrm{s}} \pi / 2 \Sigma_{\mathrm{g}}$ (with $\rho_{\mathrm{s}}$ being the volume density of a grain, with typical values of $\sim 1 \mathrm{~g} \mathrm{~cm}^{-3}$, Blum \& Wurm 2008, and $\Sigma_{\mathrm{g}}$ the local gas surface density). Particles with $S t \sim 1$ feel the strongest gas drag and therefore they move much faster to the regions of pressure maxima (e.g. Birnstiel et al. 2010; Pinilla et al. 2012). As the gas is turbulent, however, it is expected that the dust is turbulently mixed by the gas. The dust diffusion prevents the concentration of all the particles with $S t \sim 1$ inside pressure traps. The drift of particles (and hence the trapping) is efficient for particles with $S t \gtrsim \alpha_{\text {visc }}$, where $\alpha_{\text {visc }}$ is a dimensionless number that quantifies the disk viscosity (Shakura \& Sunyaev 1973). From the combination of radial drift and dust diffusion, it is expected that particles with $S t \sim 1$ are more concentrated at the pressure maximum than $S t<1$. For example, the models of dust trapping by a vortex predict that larger grains would be more azimuthally concentrated in the centre of the vortex (Birnstiel et al. 2013; Lyra \& Lin 2013), as observed in IRS 48 (van der Marel et al. 2015b) and HD 142527 (Casassus et al. 2015). Although turbulence can change the broadness of the dust concentration inside pressure bumps, it does not change the location of pressure maxima, i.e. the mean radial/azimuthal location of the dust concentration.

Figure 3 shows the model predictions for particle trapping triggered by planet-disk interaction. The gas surface density is obtained from hydrodynamical simulations of a $1 M_{\text {Jup }}$ planet embedded in the disk at $20 \mathrm{AU}$ distance from the star as in Pinilla et al. (2012). The initial gas surface density and temperature are assumed to be a power law, and the disk viscosity is taken to be $\alpha_{\text {visc }}=10^{-3}$, as found for typical values of disk turbulence in simulations of MRI-active disks (e.g. Dzyurkevich et al. 2010). The dust density distributions are obtained from dust evolution models that include the dynamics and coagulation of dust particles Birnstiel et al. (2010). When a planet opens a gap in the disk, a pressure bump is formed at the outer edge of the gap, and large particles drift to the pressure maximum located in this case at $\sim 30 \mathrm{AU}$. The particle sizes with different Stokes number $\left(S t=\left[10^{-3}, 10^{-2}, 10^{-1}, 1\right]\right)$ are shown to illustrate how the radial concentration becomes narrower for particles with higher St. The maximum grain size inside the trap is limited by dust fragmentation, which depends on $\alpha_{\text {visc }}$ (e.g. Birnstiel et al. 2010). For higher turbulence, the maximum grain size that particles can reach before fragmentation would be lower, causing the particles to easily escape from the bumps and then drift radially inwards. For more turbulent disks, a stronger pressure bump (with e.g. a more massive planet) would be required for trapping to occur (e.g. Pinilla et al. 2015). Figure 4 shows the intensity profile at 450 and $870 \mu \mathrm{m}$. The emission profile is only slightly narrower at $870 \mu \mathrm{m}$ since the range of particles sizes that are traced is comparable. Both the dust density distributions and the intensity profiles become narrower at longer times of evolution.

Because of particle trapping, the region close to the planet is empty of $\mathrm{mm} / \mathrm{cm}$ particles, and, therefore, $\alpha_{\mathrm{mm}}$ is expected to increase close to the location of the gap carved by the planet. Figure 4 also shows the expected radial profiles of $\alpha_{\mathrm{mm}}$ calculated from the models at 450 and $870 \mu \mathrm{m}$ and convolved with a 2D Gaussian profile of $35 \mathrm{AU}$ diameter. In the inner part ( $r \lesssim 10 \mathrm{AU}$ ), $\alpha_{\mathrm{mm}}$ drops because of the presence of the mmparticles. Because there is no total filtration of particles at the outer edge of the gap, small grains still flow through the gap and grow again to mm-sizes in the inner region ( $r \lesssim 10 \mathrm{AU})$. One way to prevent the presence of mm-grains is to increase the mass of the planet, and completely filter all particle sizes (Zhu et al. 2012; Pinilla et al. 2012, 2015). 

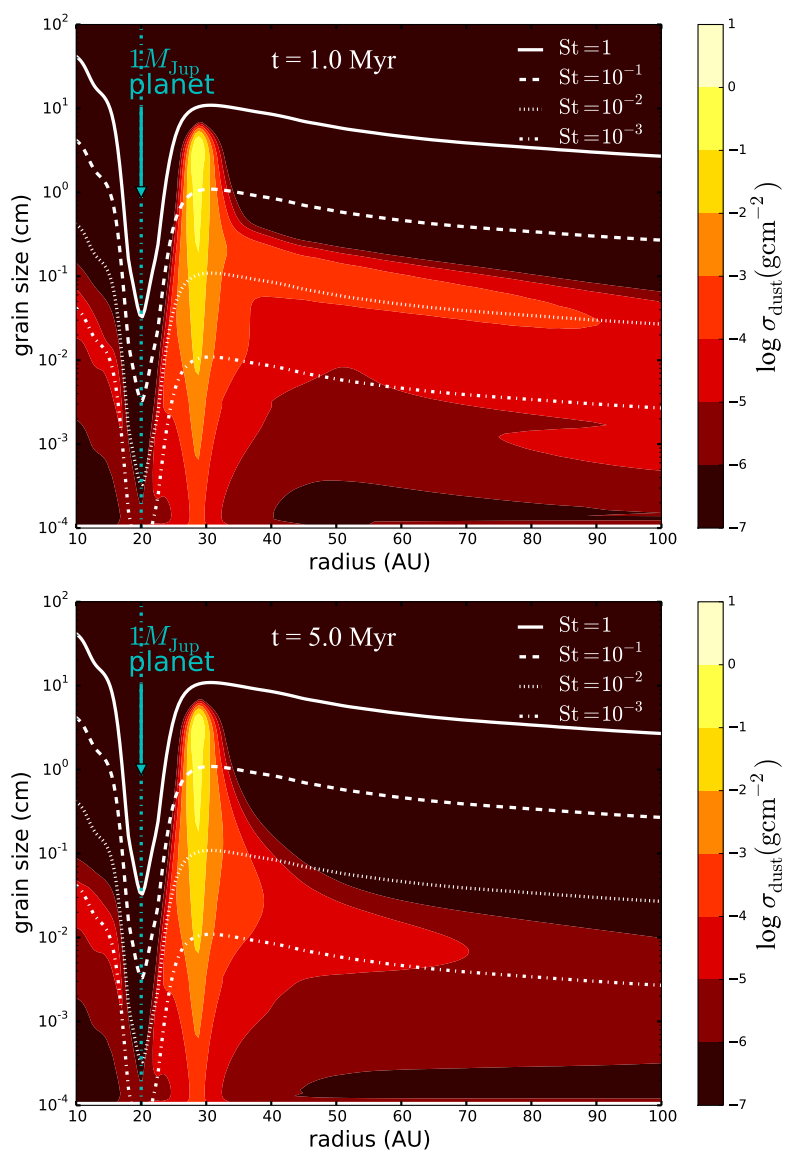

Fig. 3. Dust density distribution when $1 M_{\text {Jup }}$ is embedded at $20 \mathrm{AU}$ after 1 and 5 Myr of evolution (top and bottom panel respectively). The lines represent particle sizes with different Stokes numbers $(S t=$ $\left.\left[10^{-3}, 10^{-2}, 10^{-1}, 1\right]\right)$, which are proportional to the gas surface density profile.

At early times of dust evolution $(\sim 1 \mathrm{Myr}), \mathrm{mm} / \mathrm{cm}$ grains are still distributed in the entire outer disk $(r \gtrsim 30 \mathrm{AU})$ and radial variations of $\alpha_{\mathrm{mm}}$ are detected after convolution. This is not the case at $5 \mathrm{Myr}$, since the concentration of $\mathrm{mm} / \mathrm{cm}$ particles becomes much narrower compared to the spatial resolution and hence the potential radial variation of $\alpha_{\mathrm{mm}}$ is smeared out and is not detected.

\section{Discussion}

With the new ALMA observations of SR 21 and HD 135344B, it is possible to test whether radial/azimuthal trapping is the cause of disk structures (cavities and asymmetries). Observationally, radial trapping can be tested by analysing the location of the null in the real part of the visibilities at different wavelengths because, in the case of trapping, the mm-emission is expected to show a wider ring at shorter wavelengths. Thus, the inner edge of the ring (or dust cavity) should be located closer to the star at shorter wavelengths (Sect. 4). Additional insights can be gained by analysing the wavelength-dependent morphology and the position-dependent spectral index.

SR 21: the current observations of SR 21 at 336 and $689 \mathrm{GHz}$ suggest that the disk morphology at longer wavelengths is better described by a ring than by a vortex. From observations of ${ }^{12} \mathrm{CO}$, the disk mass of SR 21 has been inferred to be $\sim 12 \mathrm{M}_{\mathrm{Jup}}$,
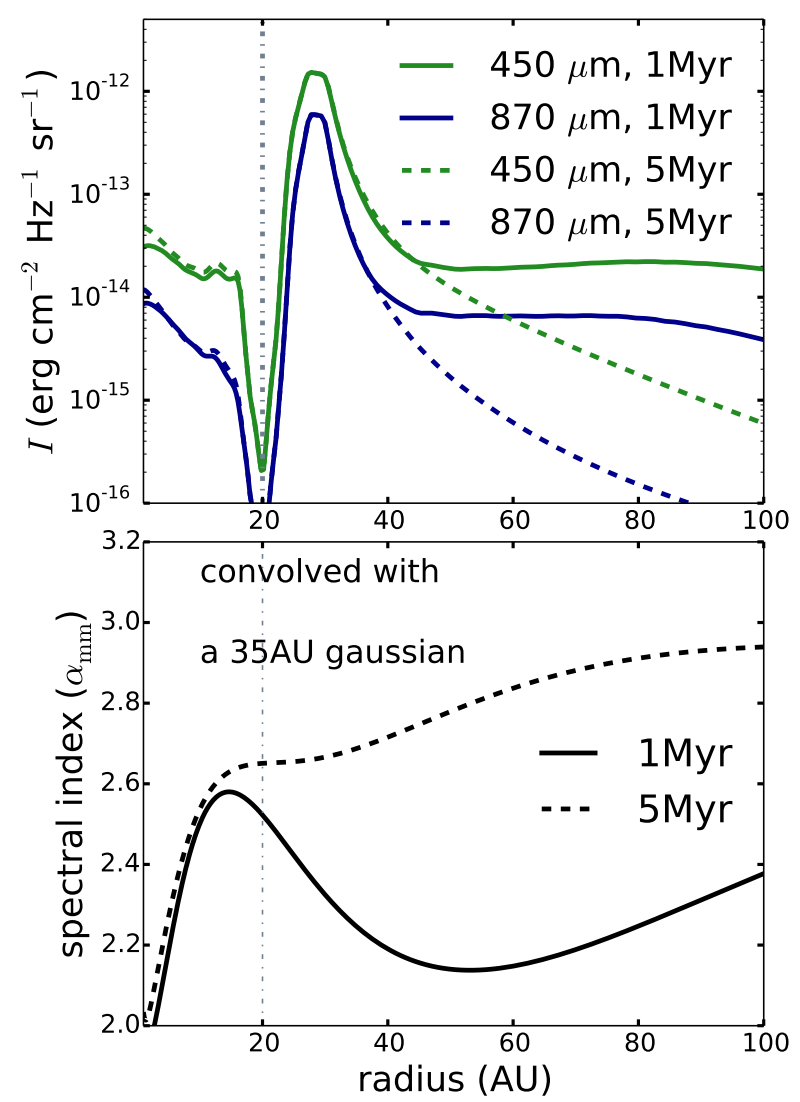

Fig. 4. Top panel: intensity radial profiles at 450 and $870 \mu \mathrm{m}$ obtained from the dust density distributions after 1 and $5 \mathrm{Myr}$ of evolution. Bottom panel: radial profile of $\alpha_{\mathrm{mm}}$ calculated from the intensity profiles at 450 and $870 \mu \mathrm{m}$, which is convolved with 35 AU Gaussian beam.

and the average value of the gas surface density close to the cavity is $\sim 80 \mathrm{~g} \mathrm{~cm}^{-2}$ (van der Marel et al. 2015a). Analysis of recent observations of ${ }^{13} \mathrm{CO}$ and $\mathrm{C}^{18} \mathrm{O}$ of this disk confirmed the disk mass (van der Marel et al. 2015c). Assuming that at each wavelength, particles with a maximum size of $\lesssim 3 \lambda$ are traced (e.g. Draine 2006), observations at Band 7 and 9 traced particles with $S t \sim 2.5 \times 10^{-3}-5.0 \times 10^{-3}$ close to the location of the cavity. These particles are still expected to be affected by radial drift and to move towards pressure maxima.

The fact that at a longer wavelength the morphology is better described by a ring than a vortex is in contradiction with model predictions of trapping by a vortex. Thus, the observed azimuthal structure is likely not caused by a vortex. Moreover, to sustain a long-lived vortex, the radial width of the vortex cannot be much higher than the disk scale height $(\lesssim 2 h$, Barranco $\&$ Marcus 2005) and the fact that the radial width of the vortex in SR 21 is much larger that the disk scale height $(\sim \times 5 h$ van der Marel et al. 2015a), as found from the morphology fitting of the Band 9 data (Table 2), also disfavours the vortex scenario.

In the radial direction, larger grains traced at $870 \mu \mathrm{m}$ are more narrowly concentrated than smaller grains traced at $450 \mu \mathrm{m}$ in SR 21, as demonstrated in the shift of the nulls of the visibilities (see Fig. 2 and Table 2). The narrower concentration of larger grains is in agreement with particle trapping by a radial pressure bump. From measuring the broadness of the dust concentration inside the trap at different wavelengths, the disk turbulence could be estimated (broader implying higher turbulence) if the disk temperature and gas surface density profile are well constraint from observations. Further evidence that radial 
trapping occurs in SR 21 is given by the radial changes of the spectral index $\alpha_{\mathrm{mm}}$, which increase outwards, as expected from radial trapping models (Fig. 4). The detection of radial variation of $\alpha_{\mathrm{mm}}$ with the current resolution suggests that particle trapping in SR 21 has occurred in $\lesssim 1$ Myr because at longer times variations of $\alpha_{\mathrm{mm}}$ would not be detected (Fig. 4). The discrepancy between model predictions and observations for the values of $\alpha_{\mathrm{mm}}$ in the inner part of the disk come from the fact that we do not have total filtration of particles in the outer edge of the gap in our model.

Another potential explanation for variations of $\alpha_{\mathrm{mm}}$ is high optical depth, in which case the physical temperature is expected to be close to the brightness temperature. To reproduce the observed differences in $\alpha_{\mathrm{mm}}$ with optical depth effects alone, the emission must originate from a small surface area. Assuming that the emission is distributed in a homogeneous ring whose temperature is equal to the highest brightness temperature at the peak of emission obtained at the two frequencies $\left(T_{B} \approx 32 \mathrm{~K}\right.$ in Band 9 and $\approx 13 \mathrm{~K}$ in Band 7 ), the ring must be very narrow ( $\lesssim 3 \mathrm{AU}$ in width). This contradicts the observed spatial extent of the emission in both bands (30-40 AU for SR 21 and 60-70 AU for HD 135344B, see Table 2). Thus, the radial variations of $\alpha_{\mathrm{mm}}$ cannot be due to optically thick emission alone. Pérez et al. (2014) demonstrated that the emission at Band 9 is marginally optically thick at the peak of emission, so it can only trace some of the mass surface density, and hence high angular resolution observations at longer wavelength are necessary to confirm our predictions at the location of the peak.

HD 135344B: in this case, the model with the best-fit parameters for the disk morphology at the two wavelength is the vortex model (Table 2). The disk mass inferred from ${ }^{12} \mathrm{CO},{ }^{13} \mathrm{CO}$, and $\mathrm{C}^{18} \mathrm{O}$ is $\sim 20 M_{\text {Jup }}$ (van der Marel et al. 2015a,c) and the average value of the gas surface density at the location of the dust cavity is similar to that in SR $21\left(\sim 80 \mathrm{~g} \mathrm{~cm}^{-2}\right)$. Therefore, the current observations trace particles with similar Stokes numbers as SR 21.

From the morphological models, the azimuthal width of the vortex increases at longer wavelengths in contradiction with predictions of particle trapping by vortices. Similar to SR 21, the radial width of the vortex is too large compared to the scale height of the disk. The origin of the azimuthal asymmetry is inconsistent with a vortex and may be related to the spiral arms observed at scattered light as also suggested by Pérez et al. (2014). In particular the bright spiral in the south coincides with the location of the asymmetry in the millimetre (Garufi et al. 2013; Quanz 2015, see also Appendix B). Indeed, the azimuthal shift of the peak (Fig. 1, also observed for SR 21) may be related to the physical rotation of spiral arms, but higher angular resolution observations are needed to confirm this prediction.

In contrast with SR 21, neither a shift of the null of the visibilities nor radial variations of $\alpha_{\mathrm{mm}}$ are detected for HD 135344B, but this does not exclude radial particle trapping. From the model predictions (Sect. 4), the dust density distribution of mm-grains is more narrowly concentrated at the pressure maximum after $5 \mathrm{Myr}$ than at $1 \mathrm{Myr}$, and radial variations in $\alpha_{\mathrm{mm}}$ would not be detected with the current resolution (Fig. 4). Hence, the fact that radial changes in $\alpha_{\mathrm{mm}}$ are not detected for HD 135344B can be related to the fact that any instability (e.g. planet) responsible for the trapping formed earlier ( $\sim \mathrm{Myr}$ ago) in HD 135344B than in SR 21 ( 1 Myr ago). Another possibility is that trapping happens in more refined structures, such as spiral arms in gravitationally unstable disks, in which case any variations of $\alpha_{\mathrm{mm}}$ remains unresolved (Dipierro et al. 2015).

Besides longer evolution times, other disk and planet parameters, such as planet mass, turbulence, or disk temperature, can also affect the gap shape and thus the radial concentration of $\mathrm{mm} / \mathrm{cm}$-sized particles in pressure bumps, leading to a different spatial distribution of small and large grains. High contrast asymmetries have been observed in other transition disks and interpreted as vortices (e.g. Oph IRS 48, van der Marel et al. 2013). Detection of vortices in transition disks may be atypical because several parameters, such as strong turbulence or feedback from dust to the gas, can prevent a vortex from being long-lived (e.g. Ataiee et al. 2013; Zhu \& Stone 2014; Raettig et al. 2015). Even in the case where a planet originally triggered the formation of the vortex, an eccentric orbit or the presence of addition planets can also lead to its rapid destruction (Ataiee \& Pinilla 2015).

The current ALMA observations of SR 21 and HD 135344B suggest that anti-cyclonic vortices are not the origin of their low contrast azimuthal asymmetries. Observations at high angular resolution at longer, optically thin wavelengths, which provide information about the distribution of larger grains, will further constrain the origin of the observed dust structures in transition disks.

Acknowledgements. We are thankful to the anonymous referee, who helped to improve the quality of this manuscript. We are grateful with M. Benisty and C. P. Dullemond for fruitful discussions. Astrochemistry in Leiden is supported by the Netherlands Research School for Astronomy (NOVA), by a Royal Netherlands Academy of Arts and Sciences (KNAW) professor prize, and by the European Union A-ERC grant 291141 CHEMPLAN. T.B. acknowledges support from NASA Origins of Solar Systems grant NNX12AJ04G. This paper makes use of the following ALMA data: ADS/JAO.ALMA\#2012.1.00158.S and \#2011.0.00724.S. ALMA is a partnership of ESO (representing its member states), NSF (USA), and NINS (Japan), together with NRC (Canada) and NSC and ASIAA (Taiwan), in cooperation with the Republic of Chile. The Joint ALMA Observatory is operated by ESO, AUI/NRAO and NAOJ.

\section{References}

Andrews, S. M., Wilner, D. J., Espaillat, C., et al. 2011, ApJ, 732, 42 Ataiee, S., Pinilla, P., Zsom, A., et al. 2013, A\&A, 553, L3

Ataiee, S., \& Pinilla, P. 2015, MNRAS, submitted

Barranco, J. A., \& Marcus, P. S. 2005, ApJ, 623, 1157

Birnstiel, T., Dullemond, C. P., \& Brauer, F. 2010, A\&A, 513, A79

Birnstiel, T., Dullemond, C. P., \& Pinilla, P. 2013, A\&A, 550, L8

Blum, J., \& Wurm, G. 2008, ARA\&A, 46, 21

Brauer, F., Dullemond, C. P., \& Henning, T. 2008, A\&A, 480, 859

Brown, J. M., Blake, G. A., Dullemond, C. P., et al. 2007, ApJ, 664, L107

Brown, J. M., Blake, G. A., Qi, C., et al. 2009, ApJ, 704, 496

Casassus, S., Wright, C., Marino, S., et al. 2015, ApJ, 812, 126

de Juan Ovelar, M., Min, M., Dominik, C., et al. 2013, A\&A, 560, A111

Dipierro, G., Pinilla, P., Lodato, G., \& Testi, L. 2015, MNRAS, 451, 5493

Dodson-Robinson, S. E., \& Salyk, C. 2011, ApJ, 738, 131

Dong, R., Rafikov, R., Zhu, Z., et al. 2012, ApJ, 750, 161

Draine, B. T. 2006, ApJ, 636, 1114

Dzyurkevich, N., Flock, M., Turner, N. J., Klahr, H., \& Henning, T. 2010, A\&A, 515, A70

Finkbeiner, D. P., Davis, M., \& Schlegel, D. J. 1999, ApJ, 524, 867

Flock, M., Ruge, J. P., Dzyurkevich, N., et al. 2015, A\&A, 574, A68

Follette, K. B., Tamura, M., Hashimoto, J., et al. 2013, ApJ, 767, 10

Garufi, A., Quanz, S. P., Avenhaus, H., et al. 2013, A\&A, 560, A105

Guilloteau, S., Dutrey, A., Piétu, V., \& Boehler, Y. 2011, A\&A, 529, A105

Høg, E., Fabricius, C., Makarov, V. V., et al. 2000, A\&A, 355, L27

Isella, A., Pérez, L. M., Carpenter, J. M., et al. 2013, ApJ, 775, 30

Johansen, A., Oishi, J. S., Mac Low, M.-M., et al. 2007, Nature, 448, 1022

Klahr, H. H., \& Henning, T. 1997, Icarus, 128, 213

Loinard, L., Torres, R. M., Mioduszewski, A. J., \& Rodríguez, L. F. 2008, ApJ, 675, L29

Lyra, W., \& Lin, M.-K. 2013, ApJ, 775, 17

Makarov, V. V. 2007, ApJ, 670, 1225

Muto, T., Grady, C. A., Hashimoto, J., et al. 2012, ApJ, 748, L22 
P. Pinilla et al.: Testing particle trapping in transition disks with ALMA

Pérez, L. M., Carpenter, J. M., Chandler, C. J., et al. 2012, ApJ, 760, L17

Pérez, L. M., Isella, A., Carpenter, J. M., \& Chandler, C. J. 2014, ApJ, 783, L13

Pinilla, P., Benisty, M., \& Birnstiel, T. 2012, A\&A, 545, A81

Pinilla, P., Benisty, M., Birnstiel, T., et al. 2014, A\&A, 564, A51

Pinilla, P., de Juan Ovelar, M., Ataiee, S., et al. 2015, A\&A, 573, A9

Pontoppidan, K. M., Blake, G. A., van Dishoeck, E. F., et al. 2008, ApJ, 684 , 1323

Quanz, S. P. 2015, Ap\&SS, 357, 148

Raettig, N., Klahr, H., \& Lyra, W. 2015, ApJ, 804, 35

Regály, Z., Sándor, Z., Dullemond, C. P., \& Kiss, L. L. 2011, A\&A, 528, A93

Ricci, L., Testi, L., Natta, A., et al. 2010, A\&A, 512, A15

Rice, W. K. M., Armitage, P. J., Wood, K., \& Lodato, G. 2006, MNRAS, 373, 1619

Shakura, N. I., \& Sunyaev, R. A. 1973, A\&A, 24, 337
Testi, L., et al. 2014, in Protostars and Planets VI, eds. H. Beuther, R. Klessen, C. Dullemond, \& Th. Henning (Tucson: Univ. of Arizona Press)

van Boekel, R., Dullemond, C. P., \& Dominik, C. 2005, A\&A, 441, 563

van der Marel, N., van Dishoeck, E. F., Bruderer, S., et al. 2013, Science, 340, 1199

van der Marel, N., van Dishoeck, E. F., Bruderer, S., Pérez, L., \& Isella, A. 2015a, A\&A, 579, A106

van der Marel, N., Pinilla, P., Tobin, J., et al. 2015b, ApJ, 810, L7

van der Marel, N., et al. 2015c, A\&A, submitted

Weidenschilling, S. J. 1977, MNRAS, 180, 57

Zhang, K., Isella, A., Carpenter, J. M., \& Blake, G. A. 2014, ApJ, 791, 42

Zhu, Z., \& Stone, J. M. 2014, ApJ, 795, 53

Zhu, Z., Nelson, R. P., Dong, R., Espaillat, C., \& Hartmann, L. 2012, ApJ, 755, 6 


\section{Appendix A: Imaginary part of the visibilities}

Figure A.1 shows the imaginary part of the visibilities at $689 \mathrm{GHz}(\sim 450 \mu \mathrm{m}$, Band 9$)$ and at $336 \mathrm{Gz}(\sim 870 \mu \mathrm{m}$, Band7) for SR 21 and HD 135344B. Non-zero imaginary visibilities indicate the presence of an azimuthal asymmetry (e.g. Isella et al. 2013). For HD 135344B, there are significant variations from zero at both frequencies. For SR 21, the non-zero values are marginal in Band 7, but are significant in Band 9 data.

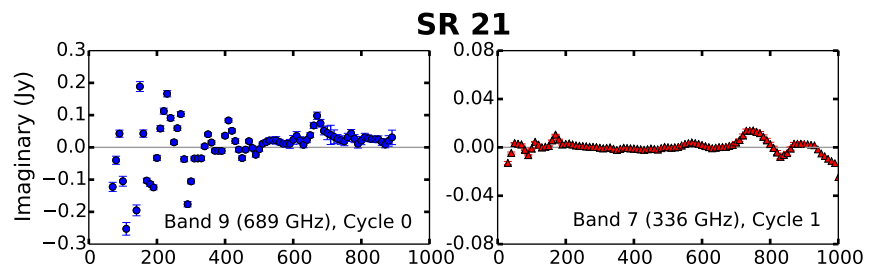

HD 135344B

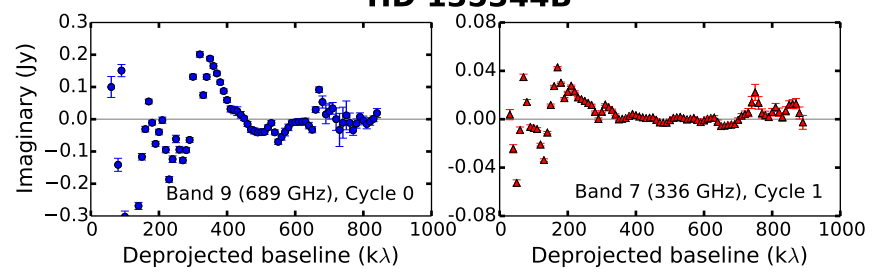

Fig. A.1. Imaginary part of the visibilities at $689 \mathrm{GHz}(\sim 450 \mu \mathrm{m}$, Band 9) and at $336 \mathrm{GHz}(\sim 870 \mu \mathrm{m}$, Band7) for SR 21 (top panel) and HD 135344B (bottom panel).

\section{Appendix B: Residual maps}

Figure B.1 illustrates the residual maps for both sources after subtracting the best-fit models (Table 2) to the Band 7 data ( $336 \mathrm{GHz}$ ). For SR 21 the best-fit is described by a ring model, whereas for HD 135344B it is a vortex model. The residuals for HD 135344B show a spiral structure, as also suggested by Pérez et al. (2014) in Band 9 (689 GHz).

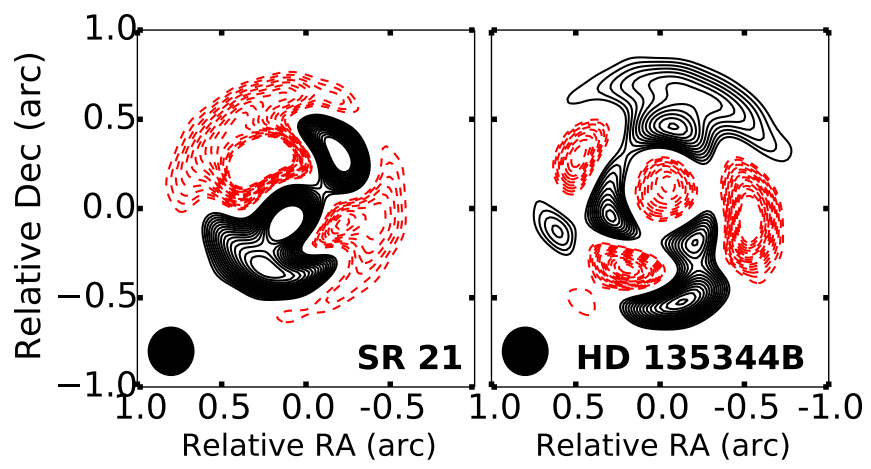

Fig. B.1. Residual maps after subtracting the best-fit models from Table 2, contours start at $\pm 3 \sigma$ and space by $3 \sigma$ steps.

\section{Appendix C: Uncertainties in the azimuthal variations of the spectral index}

The calculation of the spectral index $\left(\alpha_{\mathrm{mm}}\right)$ from the observations depends considerably on how the two images are
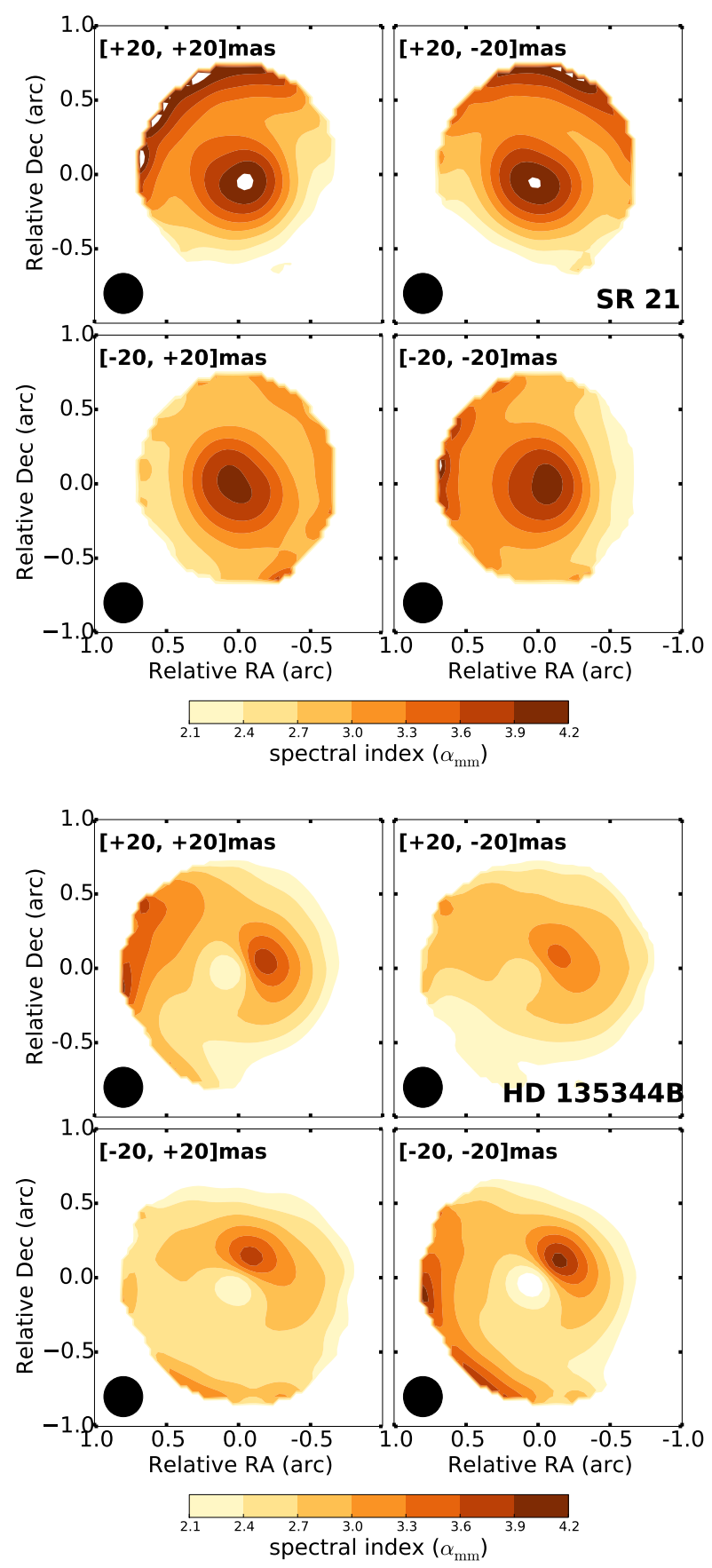

Fig. C.1. Calculated spectral index $\left(\alpha_{\mathrm{mm}}\right)$ assuming different alignments of the images for SR 21 (top panel) and HD 135344B (bottom panel).

overlapped. To demonstrate that the apparent azimuthal variations remain uncertain, we shifted the alignment of the Band 7 and Band 9 images of SR21 and HD135344B by the positional uncertainty. Figure C. 1 shows $\alpha_{\mathrm{mm}}$ by shifting 20 mas in both vertical and horizontal direction. The only reliable variation of $\alpha_{\mathrm{mm}}$ is in the radial direction for SR 21, which remains significant independent of the alignment. In HD 135344B, there is a hint of high values of $\alpha_{\mathrm{mm}}$ opposite to the azimuthal asymmetry, which also remains independent of the alignment. 